Amazonía Peruana, Volumen XVII, №34, 2021; pp. 253-266

\title{
NO UNO SINO VARIOS CASTELLANOS AMAZÓNICOS
}

\section{Alberto Chirif}

alberto.chirif@gmail.com

\section{Resumen}

El castellano amazónico sigue siendo una realidad poco estudiada. Sobre la base de dos elementos del habla característica de una parte de la población, la entonación y la alteración sintáctica, se ha construido un estereotipo sobre el habla de la región. Esto no pasa de ser una broma porque se exagera la entonación y se realizan inversiones silábicas que crean figuras inexistentes en el habla real de la región. El otro problema es suponer que en toda la región amazónica peruana se habla de la misma manera. Esto tiene que ver con la tendencia a la simplificación, como aquella que dividía el territorio peruano en tres regiones naturales. La Amazonía, que comprende alrededor del $70 \% \mathrm{del}$ territorio peruano, no es homogénea ni ambiental ni socialmente, porque ha estado y está sujeta a diferentes procesos sociales. La interacción de estos procesos con el sustrato de cada zona es lo que determina las variantes en el castellano.

Palabras claves: Amazonía; Castellano; Quechua 


\section{Summary}

Amazonian Spanish remains an under-studied reality. Based on two elements of speech characteristic of a part of the population, intonation and syntactic alteration, a stereotype has been built on the speech of the region. This is not a joke because the intonation is exaggerated and syllabic reversals are made that create non-existent expresiones in the real speech of the region. The other problem is to assume that tin the whole of the Peruvian Amazon region is spoken in the same way. This has to do with the tendency to simplification, such as the one that divided Peruvian territory into three natural regions. The Amazon, comprising about $70 \%$ of the Peruvian territory, is not homogeneous or environmental not socially because it has been and is subject to different social processes. The interaction of these processes with the substrate of each zone is what determines the variants in Spanish.

Keywords: Amazon; Spanish; Quechua

\section{Introducción}

En el presente artículo intentaremos bosquejar de manera general los diversos procesos de colonización que se han producido en la Amazonía peruana desde la Colonia y durante la República, y cómo el castellano ha sido modificado en cada zona, en mayor o menor grado, por la influencia de las culturas y lenguas indígenas prexistentes y de las lenguas de los inmigrantes. Se conoce algo sobre dicha modificación en las regiones de Loreto, Ucayali y Amazonas (aunque falta mucho por estudiar), pero nada en las otras regiones amazónicas del Perú; estas últimas no han sido objeto de atención por parte de los investigadores. En este momento no estamos en posibilidad de presentar más que algunas pistas generales que son producto de una consulta reciente realizada con diversos profesionales, de una reflexión basada en nuestra propia experiencia y de la revisión de fuentes escritas. ${ }^{1}$

Además de las mencionadas consultas, hemos revisado dos tipos de documentos escritos. Por un lado, trabajos que dan cuenta de la expansión del quechua en diversas zonas de la Amazonía peruana. La influencia de esta lengua en el castellano hablado en esta región es la que ha sido más estudiada

1 Para todas esas personas, mi sincero agradecimiento: a la lingüista Rosa Vallejo, al antropólogo Thomas Moore, a la educadora Elena Burga, al lingüista Roberto Zariquiey, a la educadora y magister en antropología Selva Chirif y al estudiante de doctorado en lingüística Alonso Vásquez. 
hasta el momento por diversos autores; entre ellos Alfredo Torero y Gerald Taylor. Por otro lado, informes de misioneros jesuitas y franciscanos de los siglos XVII y XVIII para buscar la temprana presencia en la región amazónica de palabras quechuas.

En este trabajo presentaremos a grandes rasgos los procesos de inmigración y colonización realizados en tres grandes zonas de la Amazonía peruana que hemos definido de la siguiente manera: norte, comprende el actual departamento de Loreto, y las provincias de Chachapoyas, Bagua, Condorcanqui (Amazonas), Moyobamba y Lamas (San Martín); central, incluye el actual departamento de Ucayali y las provincias de Oxapampa (Pasco), Chanchamayo, Satipo (Junín), Pachitea, Puerto Inca (Huánuco) y La Convención (Cusco); y sur, comprende el departamento de Madre de Dios.

\section{Resultados y discusión}

\section{Zona norte}

Las lenguas más habladas en la época prehispánica en esta zona pertenecieron a las familias lingüísticas tupí (kukama, kukamiria, omagua y yurimaguas), jíbaro (awajun, wampis, achuar, shuar, candoshi y xiviar) y záparo. Durante la Colonia y la República, el panorama cambió radicalmente. Las lenguas yurimaguas y omagua desaparecieron, aunque en la comunidad de San Joaquín de Omaguas existen aún algunas personas mayores que hablan esta última, mientras que el kukama y el kukamiria, aunque no son usadas en la comunicación cotidiana, sí se conservan en espacios reducidos, integrados especialmente por personas de avanzada edad y algunos jóvenes que han aprendido de ellas. Existe además un movimiento social, con influencia en comunidades y en la ciudad de Nauta, que impulsa la recuperación del kukama.

De acuerdo a la lingüista Rosa Vallejos, es difícil establecer una "fecha exacta para determinar la desaparición del kukama, pues ha sido, o sigue siendo, un proceso gradual”. Señala que las crónicas parecen indicar que, en las reducciones, el kukama fue la lengua franca. "De haber sido así, la versión de que esta lengua comenzó a desaparecer a la llegada de los conquistadores y misioneros no sería exacta. En otras palabras, si el kukama cumplió este rol, de alguna manera esto debió convertirla en algo deseable y necesario de aprender por parte de personas de otras identidades indígenas que vivieron en las reducciones. Sin embargo, esto parece no haberle otorgado a los Kukama ventajas sobre los otros pueblos, pues fueron tan oprimidos como ellos". Vallejos opina que se puede afirmar "con seguridad, [que] hacia finales 
del siglo XIX, si no antes, un buen número de cocamas ya hablaba castellano". Cita ella una afirmación en este sentido del padre Lucas Espinosa, en su libro publicado en 1935: "Los representantes de esta raza presentan un sinnúmero de intérpretes bastante familiarizados con el habla española" (Espinosa 1935: 7). Se refiere, por último, a la opinión de don Víctor Yuyarima y de doña Rosa Amías, con quienes ella trabajó temas lingüísticos, que la escuela aceleró el proceso de pérdida de la lengua (com. per. 17/3/09, cit. en Chirif 2016: 29). Con relación a esta afirmación debemos señalar dos cuestiones. La primera es que la fundación de escuelas en los caseríos habitados por población kukama en el curso bajo de los ríos Marañón y Ucayali, que por lo general eran fundos de patrones que empleaban indígenas como mano de obra, ocurrió más temprano que en otras zonas, donde recién comenzaron a establecerse en la década de 1950. De una muestra de once caseríos en el primero de esos ríos (actualmente comunidades nativas), las escuelas de seis de ellos fueron fundadas durante las décadas de 1930 y 1950; y en el segundo, de una muestra de nueve caseríos, en ocho de ellos las escuelas comenzaron a funcionar entre 1920 y 1950 (Chirif 2003: 31-38). Todas esas escuelas fueron monolingües, y esta es la segunda diferencia con las demás zonas: en las comunidades kukama nunca funcionó el sistema de escuelas bilingües impulsado por el Estado, mediante el convenio que suscribió con el Instituto Lingüístico de Verano en 1950.

En el territorio de lo que hoy constituye la actual región de Loreto, el quechua está presente desde la Colonia, pero no como lengua hablada por grupos indígenas, sean inmigrantes u otros que la pudieran haber adoptado, sino como vocablos sueltos para denominar flora, fauna, ríos, montes y demás accidentes geográficos y fabricaciones culturales. En su introducción a la obra del misionero jesuita Manuel Uriarte, que da cuenta de su labor realizada durante la segunda mitad del siglo XVIII, Constantino Bayle señala, citando fuentes referidas a ese tiempo, que en Maynas existía 40 lenguas diferentes, según unos, y 27, de acuerdo a otros. Al detallarlas, no se menciona el quechua (Uriarte 1986, n. 5: 22). Lo mismo ocurre con otros jesuitas que trabajaron en la provincia de Maynas durante ese tiempo (Figueroa 1986; Maroni 1988; Veigl 2006). No obstante, en todas estas obras se encuentra una gran cantidad de vocablos para nombrar especies de la fauna: wapo, siquisapa, upapisco, yacumama, yanac curo, rumi challua (corvina), ninacuru, guacamayo, casha cushillo, coto (que designa tanto al mono aullador como a la enfermedad del bocio); de la flora: uchu, chonta, ayahuasca, chirimoya, huacrapona, sachapapa, caspi; y accidentes geográficos: ríos Chingana y Ampiyacu, cerro Cachi Urco y Pucaurquillo, pongo, muyuna, entre otros muchos (Para significado de estos vocablos, consultar Chirif 2016) 
Como lengua hablada por grupos humanos aparece recién en la escena lingüística de esta zona entre fines del siglo XIX e inicios del siglo XX. Actualmente, constituye la lengua indígena con mayor cantidad de hablantes en ella y la de mayor influencia en la formación del castellano amazónico dominante. Es una lengua también importante en las regiones de Loreto, San Martín y Amazonas, aunque en estas apareció por procesos diferentes, como veremos más adelante.

Si observamos el mapa lingüístico de Loreto, se hace evidente que los ríos con mayor presencia de población hablante del quechua son aquellos que tienen su origen en Ecuador, donde la población adquirió la lengua en el siglo XVII o incluso antes. De noreste a suroeste, esos ríos son: Napo, Tigre y Pastaza. El caso del Putumayo, donde también existe hablantes del quechua, es diferente, ya que se trata de un proceso posterior de reemigración a partir del Napo que luego se extendió hacia el Caquetá en Colombia.

¿Cómo llegó la lengua quechua a esas regiones? Alfredo Torero ha señalado que fue a través de lo que él ha denominado "comercio lejano". Se trata de un proceso que llevó varios siglos, durante los cuales esta lengua se difundió desde la costa sur del Perú, el señorío de Chinchay, primero hacia las costas de Ecuador y más tarde hacia las alturas andinas y el Oriente. El quechua, señala este autor, se había afianzado en la costa norte y sur del actual Perú con la importancia que había cobrado la ciudad de Pachacamac como lugar santo y de peregrinación. A inicios del siglo XI, el comercio interandino entre regiones tan distantes como el Altiplano del Collao y el Ecuador tomó especial fuerza. En ese momento surgió la ciudad de Chincha para cumplir el papel de nexo entre el sureste andino y los reinos y señoríos de las costas ecuatorianas. Fue en esa dinámica que "el quechua chinchay empezó a penetrar en regiones muy distantes como lengua de relación adoptada por los señores y los mercaderes interesados en el comercio interregional andino y respaldada por el poder económico de Chincha y el ascendiente religioso y plítico de Pachacamac" (Torero 1984: 372).

Afirma Torero que, siglos más tarde, los incas trataron de poner fin al comercio impulsado por esos comerciantes, logrando solo disminuir su importancia, pero no anularlo del todo. Señala también que el quechua se estableció en el Oriente ecuatoriano a más tardar en el siglo XVI, pero se pregunta acerca "de si estaba ya en el Ecuador cuando llegaron los incas”. (1984: 375). Más adelante responde esta pregunta: "la presencia en el nororiente peruano de dialectos igualmente IIB como los de Lamas (San Martín) y Chachapoyas (Amazonas), cercanos tanto al de la costa central peruana y al de Huarochirí, hoy extintos, cuanto a las hablas ecuatorianas, refuerza la hipótesis de que la difusión de los IIB 
septentrionales de Ecuador y Perú ocurrió como parte de un fenómeno de fuerte relación económica interregional que venía desplegándose en los Andes desde siglos antes de la expansión del Imperio cusqueño" (Torero 1984: 379).

Implantado el quechua en las partes altas de los ríos ecuatorianos, el paso siguiente fue su imposición sobre poblaciones indígenas que hablaban otras lenguas. De esta manera, esa lengua se afianzó en las zonas de Tena, Baeza, Quijos y otras. Si bien durante la Colonia los jesuitas quisieron imponer el quechua como lengua franca, y con esta finalidad crearon incluso una escuela en Quito, es probable que su impacto no haya sido muy significativo considerando el poco tiempo - apenas unos 130 años - que tuvieron para actuar, antes de su expulsión en 1767. Por lo demás, ninguna lengua se implanta por enseñanza en las aulas sino por su difusión social. En este sentido es más verosímil que su difusión y arraigo haya sido consecuencia del comercio lejano y de procesos migratorios.

Pero el comercio lejano no solo se daba entre poblaciones que hablaban quechua. Según señala Torero (1984: 380): "Posiblemente desde varios siglos antes de la conquista hispana, gentes de habla quechua habían entrado en contacto con los omagua-cocama en el nororiente ecuatoriano y el nororiente peruano y con uno o varios pueblos de Colombia, cuyas lenguas tomaban el relevo como idiomas de relación para el comercio lejano". El intercambio debe haber sido fuerte, como lo indica la existencia de palabras comunes en los vocabularios del quechua y del kukama.

Con relación a la aparición de población quechua hablantes en la parte peruana de los ríos que descienden de Ecuador (Pastaza, Corrientes, Tigre y Napo), si bien pueden haber existido procesos migratorios independientes y a pequeña escala anteriores, existen evidencias de que esta se produjo recién durante el cambio de siglo XIX-XX, como consecuencia de la acción de los patrones explotadores de gomas silvestres, que necesitaban mano de obra. Así lo afirman Villarejo y Mercier para el caso de los quechuas del Napo y Tigre (cit. en Ribeiro y Wise 1978: 161 -167), quienes indican además que su llegada significó la absorción de grupos indígenas disminuidos por las epidemias, como los Aushiri, los Taushiro, los Záparo (y sus múltiples variantes: Andoa, Arabela e Iquito, entre otros). Antes, ellos habían impuesto el quechua "en la federación de los Quijos, Santarrosinos, etc., durante 4 siglos de contactos socio-económico" (Ribeiro y Wise 1978: 161).

En los ríos Pastaza, Corrientes y Tigre se produjo además un proceso de intercambio matrimonial entre quechuas y achuares, por lo que es común 
encontrar los mismos apellidos en unos y otros (Dahua, Sandi y Hualinga, por ejemplo), y muchas personas bilingües. (Guzmán-Gallegos 2017: 42-43).

El origen de la presencia de esta lengua en Amazonas y San Martín, según el lingüista Gerald Taylor, es anterior a la Colonia y parece estar relacionada con la presencia de los incas que conquistaron a los Chachapoyas durante la segunda mitad del siglo XV. Los estudios lingüísticos indican que las características del quechua de San Martín y Amazonas son semejantes. Taylor señala que: "Según la tradición, la introducción del quechua en los territorios de la antigua 'federación de los Chachapoyas' fue obra de los incas quienes, conforme a su política habitual, hicieron educar a los nobles chachas en el Cuzco y organizaron la administración de su provincia según las normas del estado inca" (Taylor 2000: 31). La influencia de los incas en la zona del Huallaga también es puesta de manifiesto por la extensa red de caminos antiguos que fueron usados por la expedición de Ursúa y Lope de Aguirre en búsqueda de El Dorado, durante el siglo XVI. En Amazonas, el quechua fue modificado bajo el influjo de la lengua original de los habitantes, ahora totalmente perdida. De ella solo han quedado ciertos topónimos y apellidos, como Chuquipiondo, Valqui, Soplín, Guabloche, Huarote y otros (Ibíd.: 27-30). También el quechua ha desparecido casi por completo en esta región y está ahora reducido a unas pocas comunidades. En San Martín, la población de habla quechua se concentra actualmente en dos provincias, Lamas y Tabalosos. La lengua se encuentra muy activa en la primera de ellas. En los dos departamentos, sin embargo, es incuestionable su importancia en la formación del castellano regional, considerando que gran parte del poblamiento de Loreto, incluido Ucayali con quien conformaba una unidad política hasta inicios de la década de 1980, se hizo a partir de inmigrantes de San Martín.

En el proceso de la formación del castellano regional, tan importante como la castellanización de los kukamas o la llegada del quechua desde Ecuador, es la inmigración hacia Loreto de población sanmartinense, sea quechuahablante o castellanohablante, porque en este segundo caso tiene que haber sido un castellano ya influido por el quechua durante la Colonia y cerca de un siglo de República. Es importante que señalemos que estos inmigrantes no eran "colonos" (como los andinos que se desplazan hacia la Amazonía, desde la segunda mitad del siglo XIX en búsqueda de tierras), sino personas de cultura amazónica. Este hecho es lo que permitió su adaptación sin mayor problema a la parte baja de la cuenca y su mestizaje, biológico y cultural, con la población local. En ese proceso ha surgido un nuevo sector social, llamado "ribereño" o "mestizo ribereño" o "campesino ribereño", que asimismo está integrado por personas 
indígenas (principalmente kukamas, aunque también shawis, shiwilus y otros) que buscan ocultar su identidad en su intento de escapar al desprecio racista. Es un sector muy especial y cambiante, ya que, como ha quedado demostrado desde la década de 1980, muchos calificados como mestizos ribereños han comenzado a reconocer sus orígenes indígenas, que hasta entonces mantenían soterrados, en un esfuerzo de escapar de las presiones racistas ejercidas por la sociedad. Por este proceso de retorno a su identidad indígena han transitado numerosos kukamirias, kukamas y shiwilus, principalmente. En otras palabras, ellos han dejado de autonombrarse como "ribereños" para recuperar la identidad de sus ancestros e incluso para tratar de reaprender las lenguas de ellos.

Hemos consultado con diversas personas acerca de las diferencias que han notado en el habla del castellano loretano más característico con el de otras provincias incluidas en esta zona norte, como Chachapoyas, Ucayali, Yurimaguas o Caballo Cocha. Todas han señalado que sí existen diferencias, aunque no están en capacidad de detallarlas, dado que el tema no ha sido motivo de sus investigaciones. En este sentido, sus apreciaciones deben ser tomadas solo como indicadores de variedades del castellano.

La lingüista Rosa Vallejo (com. per. 1-2/7/20) señala que en su trabajo ha planteado que "la hipótesis que la dicotomía rural/urbana no captura correctamente lo que vemos en el castellano en la Amazonía”. Si bien es cierto existen "variedades urbanas (Iquitos, Yurimaguas, Nauta), la categoría rural es más complicada. Cuando nos acercamos a comunidades indígenas bilingües o multilingües, podemos notar que su castellano muestra efectos de contacto con las lenguas indígenas locales. Así, por ejemplo, el castellano que hablan los kukama es un tanto diferente al que hablan los shipibo, bora, o secoya. Las particularidades de estos castellanos se pueden explicar generalmente a partir de los rasgos de las lenguas indígenas".

Con relación al habla de Amazonas, región de la cual ella es oriunda, indica que "la entonación de ambas variedades tiene rasgos comunes, pero no son iguales". En Loreto, las vocales de las primeras sílabas suelen ser bastante alargadas, hasta más largas que las vocales acentuadas. Por ejemplo, en la palabra hermano, la vocal /e/ suele ser más larga que la /a/, que es la acentuada. Ese es uno de los rasgos que comúnmente llamamos acento charapa. Ese alargamiento vocálico inicial existe en Amazonas, pero es menor y por eso se percibe como menos marcado. En la morfosintaxis, tanto en Amazonas como en Loreto es muy frecuente el doble posesivo (su casa de mi primo), pero en Amazonas es mucho más rara la permutación del genitivo (de mi primo su casa). En Amazonas no he notado la discordancia de número (No soy sus empleada). Me parece que en Amazonas es 
más común la doble marcación de objeto (no le quiero dejar a mi hija) y el uso de artículos con nombres propios (la Rosa)".

La educadora Elena Burga (com. per. 3/7/20) señala haber notado "que los Shipibo tenían tonalidades y formas algo distintas de hablar el castellano, producto, supongo, de la influencia de su lengua materna. Ellos no hablan como el loretano o ucayalino mestizo castellano hablante; con ellos sí hay diferencia, aunque la gente de fuera no creo que lo note. Cuando fui a la zona de los kukama, a San Martín de Tipishca y a esa parte del Marañón, también noté que la tonalidad del castellano amazónico es más exagerada que en el Ucayali”.

La educadora y antropóloga Selva Chirif (com. per. 2/7/20) recoge la opinión de la señora Janet Olave Parente, del pueblo tikuna: "Hay personas en Caballococha que al hablar castellano usan palabras del tikuna y del yagua. También mezclan el habla brasileña y colombiana. En Caballococha las personas hablan diferente a Iquitos".

El estudiante de doctorado Alonso Vásquez, que realizó trabajo de campo entre los Shiwilu (Alto Amazonas), nos indica (com. per. 17/7/20) que "definitivamente hay una semejanza (ambos tipos de habla serían 'charapas'), pero intuitivamente percibo cuestiones distintas. No puedo decir más por ahora porque esto no fue objeto de mi investigación".

\section{Zona central}

Las lenguas indígenas más habladas en esta zona pertenecen a los troncos arawak y pano. Por diversos motivos, que señalaré más adelante, esta zona se mantuvo relativamente aislada de procesos migratorios y de influencias misionales como los ocurridos en la zona norte.

Jugaron un papel decisivo en favor del aislamiento del Alto Ucayali las disputas jurisdiccionales que sostuvieron jesuitas y franciscanos por el control de esa parte de la cuenca, que estuvieron a punto de desembocar en enfrentamientos de grupos de indígenas armados por dichas órdenes. Esas disputas llegaron a su apogeo cuando los franciscanos, en 1685, fundaron un pueblo habitado por unos 1800 conibos, que se ubicaba en el Ucayali cerca de la desembocadura del Pachitea y lo dedicaron a San Miguel. Sin embargo, poco después, el jesuita Heinrich Richter llegó al lugar y construyó una iglesia con campana y altar dedicados a San Francisco Javier. Un Edicto Real del 24 de abril de 1687 puso fin a estas disputas y determinó que los jesuitas tuvieran el control desde ese pueblo hacia el norte y los franciscanos hacia el sur. Las disputas entre las dos 
órdenes religiosas están referidas por Amich, quien ofrece la visión franciscana, y por Maroni y Veigl, que presentan la de sus rivales (Amich 1988: 109-114, 122; Maroni 1988: 288-289; Veigl 2006: 142-145). No obstante, el mismo año del edicto, los franciscanos resolvieron abandonar su trabajo evangélico en el Alto Ucayali, cansados por los fracasos de su labor y por las muertes de sus misioneros causadas por los indígenas, e iniciaron sus labores en la parte baja de la cuenca, a la que arribaban entrando por la ruta del Huallaga.

El trabajo de los franciscanos quedó entonces confinado a la zona de la selva de los actuales departamentos de Junín (Chanchamayo y Satipo) y Pasco (Oxapampa). Sin embargo, al promediar el siglo XVIII, nuevos obstáculos se opusieron a su labor evangelizadora en esta zona. Una serie de rebeliones - unas más importantes que otras- cerraron el paso a la actividad misionera. La más notable de ellas fue la liderada por Juan Santos Atahualpa en 1742 (Varese 1973). Como consecuencia de ella, los franciscanos fueron expulsados de la selva central y los indígenas recuperaran su libertad. En 1766, un indígena setebo llamado Runcato, se levantó en el Ucayali con el apoyo de shipibos y conibos. Catorce misioneros encontraron la muerte.

La rebelión de Juan Santos Atahualpa cerró las puertas de acceso al Ucayali por la ruta de la selva central durante casi un siglo. Todo este conjunto de hechos (rebeliones, pugnas entre las dos órdenes misioneras y menor importancia del Alto Ucayali para la administración colonial) explica por qué los indígenas asentados en esta zona pudieron mantenerse más libres de influencias externas. Pero hay un factor más que creemos importante señalar. Salvo los conibo, que eran una sociedad ribereña asentada a lo largo del Ucayali, todos los demás pueblos indígenas eran de asentamiento interfluvial, lo que implica asentamientos más dispersos y demográficamente menos densos. En los años de vigencia de las reducciones, la población obligada a vivir concentrada en determinados lugares se rebeló contra este modelo y se internó en el bosque. Esto no sucedió con los pueblos del tronco tupí de la zona norte, para quienes las reducciones no implicaron un gran cambio de sus modelos de asentamiento, porque ellos estaban acostumbrados a vivir en caseríos muy poblados.

Por otro lado, la ausencia de inmigrantes impidió que se forjase un tipo de mestizaje cultural y lingüístico, que, por cierto, de haber sucedido, el resultado hubiera sido muy diferente al de la zona norte, debido a que se hubiese realizado con colonos, es decir, con personas de cultura andina y no amazónica. No obstante, existe en esta zona un sector menor de ribereños venidos de la zona norte, que se asienta en el curso medio del Ucayali y en el Pachitea. 
Lainmigración andina hacia esta zona central recién comenzó aproximadamente cincuenta años después de fundada la República, y la relación de la población indígena con los colonos fue al comienzo muy conflictiva, por la disputa territorial, y por tanto poco adecuada para el mestizaje de culturas y lenguas. Colonos del Cuzco ocuparon tierras de los machiguengas del Alto Urubamba y colonos de Pasco y Junín invadieron las de asháninkas y yaneshas en las partes selváticas de estos departamentos. Las tensiones entre foráneos y nativos han cambiado un poco en los últimos tiempos, pero no han producido fusiones tan intensas como las de la zona norte. Al igual que en la zona norte, en esta existe también una gran cantidad de vocablos de origen quechua para animales, plantas, accidentes geográficos y productos culturales, como resultado de los intercambios entre la población local amazónica con andinos, que se remonta a fechas anteriores a la expansión de los incas (Smith 2004). Existen influencias lingüísticas recíprocas entre inmigrantes quechua hablantes (algunas ya castellanizados) y pobladores de lenguas indígenas amazónicas. Pero esto no ha sido estudiado, tal vez porque no resulta tan atractivo (en tono y vocabulario) como la de la zona norte.

El lingüista Roberto Zariquiey (com. per. 12/7/20), que realizó una investigación sobre la lengua kakataibo (pano), señala: "Estoy de acuerdo sobre la diversidad del español amazónico. El español de los kakataibo tiene una impronta pucallpina fuerte, y en pronunciación y sintaxis se parece mucho al español de Pucallpa y probablemente al de Iquitos. Sin embargo, las diferencias entre Iquitos y Pucallpa se están conociendo mejor y en ese sentido es también generalizar decir que se trata de una misma variedad. El español de los kakataibo tiene sus particularidades también y varía mucho entre hablantes de distintas generaciones. $\mathrm{El}$ verbo puede ir al final y ocurre así muchas veces. También hay patrones de discordancias de género y número y, claro, usos idiosincrásicos de preposiciones (la más común es yo voy “en” Lima), entre otros rasgos.

\section{Zona sur}

Las lenguas más habladas en esta zona pertenecen al tronco harakmbut. Adicionalmente, hay presencia de dos lenguas arawak (machiguenga y yine) y una tacana (ese eja). Si bien existen algunas comunidades de origen shipibo y quechua santarrosinos, con población trasladada por los caucheros a la zona a inicios del siglo XX, los descendientes actuales ya no hablan las lenguas de sus antepasados.

Esta zona es particular por varias razones. Desde el punto de vista geográfico, está separada del resto de la Amazonía peruana por una cordillera que divorcia 
las aguas del Madre de Dios de las del resto del sistema fluvial amazónico peruano. De esta manera, el Madre de Dios, en vez de correr hacia el norte y unir sus aguas al eje Urubamba-Ucayali-Amazonas, corre hacia el sur, y si bien tributa en el Amazonas, lo hace en Brasil después de haber pasado por Bolivia y experimentado dos cambios de nombre: Beni y Madera o Madeira. Es también particular porque la población indígena del departamento no tuvo contacto con misioneros durante la época colonial, que recién se realizó en la década de 1940, cuando los dominicos fundaron la misión de Shintuya. El contacto más significativo de indígenas con foráneos fue el realizado por Fitzcarrald, a inicios del siglo XX, cuando asesinó masivamente a indígenas con la finalidad de despejar áreas para que sus obreros pudieran extraer gomas silvestres. Durante más de un siglo después de fundada la República, Madre de Dios tampoco fue objeto de colonización andina masiva. Esta recién comenzó a hacerse sentir en la década de 1970 , y no por inmigrantes en búsqueda de tierras de cultivo, sino por extractores de oro y por trabajadores de la madera al servicio de empresas forestales. Se trata principalmente de excedentes poblacionales de Puno y Cusco, de tradición lingüística quechua y aimara, que han invadido tierras de comunidades nativas.

Desde 1970 a la fecha, Madre de Dios ha sido el departamento que más ha crecido poblacionalmente, tanto por los factores mencionados como por la construcción de la carretera Interoceánica Sur, que vincula la red vial del Perú con la de Brasil.

Con relación al tema que nos ocupa, el antropólogo Thomas Moore (com. per. 1/7/20) señala: "Entre el harakbut y el castellano regional no hay préstamos consolidados. Algunos términos que los Ministerios de Educación (DIGEIBIRA) y Cultura han difundido son repetidos a veces, pero no se han incorporado en el habla de los migrantes y no-indígenas nativos. Lo que sí es diferente del hablar loretano y ucayalino son algunos nombres de especies de flora y fauna. Por ejemplo, no se dice majaz, sino picuro. No se dice toé, sino hayapa o ayapa”.

\section{Conclusiones}

Lo señalado brevemente en este artículo nos lleva a la conclusión de que no existe un castellano amazónico sino varios, y que esto es válido incluso en la zona norte, donde el castellano tiene cierta homogeneidad lingüística. Por el momento no tenemos manera de demostrar de manera detallada en qué consisten estas variaciones y solo podemos inferirlas de la complejidad de los procesos que hemos señalado en este texto y por supuesto de las pistas que nos han dado los investigadores consultados. 
Los inicios del castellano amazónico que hoy conocemos de la zona norte son recientes. No tienen más de cien años, inicia con la implantación mayoritaria del castellano entre los kukama (Espinosa 1935) y con la llegada al Perú de quechua-hablantes de Ecuador por los ríos que tiene origen en este país. En la conformación de este castellano concurren varias lenguas y combinaciones lingüísticas. Tendemos siempre a ver los factores recientes, porque son más evidentes, pero no los componentes que han entrado en su configuración. Hablamos de la influencia del quechua, pero no sabemos cuánto de las lenguas que este subsumió en su proceso de expansión existe en ella o de las lenguas con las cuales estableció relaciones más horizontales, como el kukama o el achuar. Por otro lado, el quechua proveniente de Ecuador tampoco es homogéneo, ya que presenta al menos dos variedades, la del Napo y la del Pastaza.

El quechua se expande hacia la actual provincia de Maynas recién a partir de la época del caucho, aunque desde antes el siglo XVII se encuentran vocablos de esta lengua para nombrar elementos de la fauna, flora, accidentes geográficos y creaciones culturales. En cambio, en Amazonas y San Martín la presencia del quechua es anterior a los incas. Esta lengua ha desaparecido en el primer departamento, mientras que en el segundo continúa vigente. En ambos casos, sin embargo, es evidente su influencia en la formación del castellano, tema que merece mayor estudio.

Con relación a las otras dos zonas, no existe aún un castellano que caracterice el habla de indígenas y foráneos. El castellano ha incorporado de manera aislada algunos términos provenientes de lenguas indígenas, pero no existe una influencia grande de ellas en el habla general, ni tampoco del quechua o del aimara, más allá de las que son comunes al castellano general del Perú. Esto, sin embargo, no quiere decir que no existan otros rasgos que puedan caracterizar el castellano de esas zonas. Pero esto está por investigar.

\section{Referencias bibliográficas}

Amich, José. Historia de las misiones el Convento de Santa Rosa de Ocopa. Iquitos: Monumenta Amazonica. Centro de Estudios Teológicos de la Amazonía, Instituto de Investigaciones de la Amazonía Peruana, 1988.

Chirif, Alberto. Proyecto de apoyo organizativo. Sistematización. Iquitos: Junglevagt for Amazonas WWF-AIF/DK, 2003. 
-.Diccionario Amazónico. Voces del castellano en la selva peruana. Centro Amazónico de Antropología y Aplicación Práctica, Lluvia Editores. Lima: 2016.

Espinosa, Lucas. Los Tupi del Oriente Peruano. Madrid: Publicación de la expedición iglesias al Amazonas, 1935.

Figueroa, Francisco y Cristobal de Acuña, et al. Informes de Jesuitas en el Amazonas. 1660-1684. Iquitos: Monumenta Amazonica. Centro de Estudios Teológicos de la Amazonía, 1986.

Guzmán-Galleros, María. Nukanchi Narwpa Timputa Yarisha Tantarishun. Surcando nuestra memoria. Iquitos: Formabiap, Aidesep, Universidad de Oslo, 2017.

Maroni, Pablo. Noticias auténticas el famoso río Marañón. Iquitos: Monumenta Amazonica. Centro Estudios Teológicos de la Amazonia, 1988.

Ribeiro, Darcy y Mary Wise. Los grupos étnicos de la Amazonia peruana. Lima: Instituto Lingüístico de Verano, 1978.

Smith, Richard. "Donde nuestros ancestros alguna vez caminaron. Territorialidad y lugares sagrados Amuesha En la Amazonía Andina del Perú Central”. Ponencia presentada en el Coloquio Internacional de la Universidad de Paris III - Nueva Sorbona, Etre Indien dans les Amériques. Spoliations et résistance - Mobilisations ethniques et politiques du multiculturalisme: una perspective comparative, organizada por IHEAL-CREDAL, CEC, OPEA y CERVEPAS. París, diciembre 2-3,2004. Consultada en: https://centroderecursos.cultura. $\mathrm{pe} /$ sites/default/files/rb/pdf/Donde\%20nuestros\%20ancestros\%20alguna\%20 vez\%20caminaron\%20Territorialidad.pdf.

Taylor, Geral. Estudios lingüisticos sobre Chachapoyas. Lima: Universidad Nacional Mayor de San Marcos, Instituto Francés de Estudios Andinos, 2000.

Torero, Alfredo. "El comercio lejano y la difusión del quechua. El caso del Ecuador”. En: Revista Andina. 2 (1984): 367-402.

Uriarte, Manuel. Diario de un misionero de Maynas. Iquitos: Monumenta Amazonica. Centro de Estudios Teológicos de la Amazonia, 1986.

Varese, Stefano. La Sal de los Cerros. Lima. Lima: Retablo de Papel Ediciones, 1973.

Veigl, Franz. Noticias detalladas sobre el estado de la provincia de Maynas en América Meridional hasta el año 1768. Lima: Monumenta Amazónica, 2006. 